\title{
Quantitativer CRP-Schnelltest verbessert Prognoseabschätzung bei Blasenkrebs
}

- Das C-reaktive Protein (CRP) zeigt als Marker der Akut-Phase-Reaktion bakterielle Entzündungen an, findet sich jedoch auch bei vielen Karzinomen und ist oft mit einer Tumorprogression assoziiert, so auch bei Tumoren urothelialen Ursprungs. Bei Blasenkrebs-Patienten ist der präoperative CRPWert ein prognostischer Marker.

Jetzt steht mit "InfectCheck CRP" ein neuer CRP-Schnelltest zur Verfügung. Die quantitative Messung kann mit dem neuen mobilen Messgerät "concile $\Omega 100$ “ erfolgen.

In der urologischen Praxis ist die Ermittlung quantitativer CRP-Werte eine Hilfe für Dia- gnose und Therapiekontrolle. Bei Heilung oder erfolgreicher Therapie sinkt die CRPKonzentration innerhalb kurzer Zeit auf den Normalwert ab. Bei Patienten mit einem Harnblasenkarzinom ist der prä-operative CRP-Wert ein prognostischer Parameter und signifikant mit einem höheren Tumorstadium, Lymphknotenbeteiligung, erhöhter Lymphknotendichte und positiven Resektionsrändern assoziiert.

Eine Arbeitsgruppe um Dr. Georgios Gakis und Prof. Arnulf Stenzl an der Klinik für Urologie der Eberhard-Karls-Universität, Tübingen, wertete kürzlich retrospektiv die Daten von 246 Patienten nach Zystektomie und Lymphadenektomie aufgrund eines invasiven Harnblasenkarzinoms aus. BlasenkrebsPatienten mit präoperativ normalen CRPWerten ( $\leq 5 \mathrm{mg} / \mathrm{L}$ ) hatten eine 3-JahresÜberlebensrate von $74 \%$ und eine 5-JahresÜberlebensrate von 71,6\%. Dagegen hatten Patienten mit einem prä-operativ erhöhtem CRP-Wert nur Überlebensraten von $44 \%$ beziehungsweise $40,5 \%$.

Die CRP-Konzentration im Serum ist neben dem Tumorstadium, der Lymphknotendichte und positiven Resektionsrändern ein unabhängiger Risikofaktor und kann daher die richtige Klassifizierung von Patienten mit Blasenkrebs erleichtern.

Nach Informationen von Concile, Freiburg

\section{Testosteronsubstitution bei männlichem Hypogonadismus}

— Unabhängig vom Alter besteht ein Zusammenhang zwischen der Testosteronkonzentration und dem metabolischen Syndrom: Je mehr Komponenten des metabolischen Syndroms vorliegen, desto niedriger sind die Testosteronwerte [Haring R et al. Diab 2009; 58: 2027-31]. Der Grund dafür liege in der endokrinen Funktion der viszeralen Fettmasse, berichtete Prof. Farid Saad, Berlin. Viszerales Fett hemmt über die Freisetzung zahlreicher Mediatoren die Testosteronproduktion.

Die European Male Aging Study hat ergeben, dass die Testosteronspiegel auch im Alter annähernd stabil bleiben [Wu FCW et al. J Clin Endocrin Metab 2008; 93: 2737-45]. Unterschiede zeigten sich in Abhängigkeit vom Body-Mass-Index (BMI), wobei Männer mit einem $\mathrm{BMI}<25$ $\mathrm{kg} / \mathrm{m}^{2}$ die höchsten Testosteronwerte hatten. Mit steigendem BMI nimmt die Prävalenz eines Testosteronmangels zu. Bei BMI-Werten $\geq 35$ $\mathrm{kg} / \mathrm{m}^{2}$ haben über $60 \%$ der Männer einen Hypogonadismus [Corona G et al. J Sex Med 2008; 5: 2454-63]. Andererseits steigen unter Androgendeprivation Körperfett, Gewicht und Bauchumfang stetig an [Hamilton EJ et al. Clin Endocrinol 2011; 74: 377-83]. Diese Veränderungen treten bereits in den ersten zwölf Wochen des Testosteronentzugs auf. Eine Studie mit Testosteronundekanoat (Nebido ${ }^{\oplus}$ ) bei 184 hypogonadalen Männern mit metabolischem Syndrom zeigte neben einer Normalisierung der Testosteronwerte eine kontinuierliche Abnahme von BMI, Gewicht und Bauchumfang. Gleichzeitig wurde in der Verumgruppe eine signifikante Abnahme des CRP beobachtet, was auf einen antientzündlichen Effekt von Testosteron hinweist [Kalinchenko $S$ et al. Clin Endocrinol 2010; 73: 602-12]. Zudem verbessert die Testosteronsubstitution laut Saad depressive Symptome, Lebensqualität, sexuelle Dysfunktion und Insulinresistenz.

Abdol A. Ameri

15. Roundtable-Gespräch „Die Facetten der Testosteronwirkung beim Mann“ im Rahmen des 63. DGU-Kongresses, Hamburg,

15. September 2011

Veranstalter Jenapharm, Jena

\section{Harninkontinenz: bei Therapie- versagen Ausgangsprämisse prüfen}

- Die Frage nach dem Stellenwert alternativer Methoden bei Dranginkontinenz stellt sich laut Dr. Andreas Wiedemann, Witten/Herdecke, spätestens dann, wenn eine Anticholinergika-Therapie nicht den gewünschten Erfolg zeigt. Betrachte man die alternativen Möglichkeiten, dann zeige sich, dass viele angesichts ihres Zulassungsstatus im Routineeinsatz nicht erlaubt sind. „Anticholinergika sind nach wie vor die Grundlage der Therapie der überaktiven Blase $(O A B)$ “, erklärte der Urologe. Er empfahl, zunächst die Grundvoraussetzungen der OABTherapie zu überprüfen, diese zu optimieren sowie eine alternative Therapie für seltene Einzelfälle zu reservieren.

Bei (scheinbaren) Anticholinergikaversagern ist eine erweiterte Diagnostik indiziert, durch die auch seltene Ursachen einer symptomatischen $O A B$ ans Licht kommen. Erfragt werden sollte auch, ob ein verordnetes Anticholinergikum überhaupt eingenommen wird. Einen Grund für die Beobachtung, dass nach zwei Jahren nur noch jeder vierte Patient sein Medikament nimmt [Brostrom S et al., Eur J Clin Pharmacol 2009; 65: 309-14], sieht Wiedemann unter anderem in den typischen Nebenwirkungen ZNS-gängiger Präparate, wie beispielsweise Mundtrockenheit. Anticholinergikaversagen könne zudem durch Unterdosierung oder eine „Zytochrom-Problematik" bedingt sein.

Optimiert werden kann die Anticholinergika-Therapie zum Beispiel durch den Einsatz von Trospiumchlorid (Spasmex ${ }^{\circledast}$ ): Es ist aufgrund seiner Hydrophilie das einzige nicht ZNS-gängige Anticholinergikum. Als quartäres Amin wird Trospiumchlorid im Unterschied zu den tertiären (lipophilen) Aminen nicht hepatisch abgebaut, sondern gelangt in aktiver Form in die Harnblase und kann dort neben der systemischen auch eine lokale Wirkung entfalten.

Ute Ayazpoor

15. Bamberger Gespräche 2011 „Harninkontinenz - Was Sie schon immer wissen wollten“, 10. September 2011, Bamberg

Veranstalter: Dr. Pfleger, Bamberg 